\title{
Online Handwriting Recognition Using an Accelerometer-Based Pen Device
}

\author{
Jeen-Shing Wang \\ Dept. of Electrical Engineering \\ National Cheng Kung University \\ Tainan, Taiwan \\ jeenshin@mail.ncku.edu.tw
}

\author{
Yu-Liang Hsu \\ Dept. of Electrical Engineering \\ National Cheng Kung University \\ Tainan, Taiwan \\ yulianghsu@mail.ncku.edu.tw
}

\author{
Cheng-Ling Chu \\ Dept. of Electrical Engineering \\ National Cheng Kung University \\ Tainan, Taiwan \\ bzero0122@hotmail.com
}

\begin{abstract}
This paper presents an accelerometer-based pen device for online handwriting recognition applications. The accelerometer-based pen device consists of a triaxial accelerometer, a microcontroller, and an RF wireless transmission module. Users can hold the pen device to write numerals in air without space limitations. The accelerations generated by hand motions are generated by the accelerometer embedded in the pen device, and are transmitted to a personal computer for further signal preprocess via the wireless module. Subsequently, a dynamic time warping (DTW) algorithm is applied to align the accelerations and search class templates for each digit in the training stage. Finally, the accelerations can be recognized via the alignment with the class templates in the testing stage. The average user-dependent and userindependent recognition rates were $90.6 \%$ and $84.8 \%$, respectively. Our experimental results have successfully validated the effectiveness of the accelerometer-based pen device for 3-dimensional (3-D) online handwriting recognition.
\end{abstract}

\begin{tabular}{|c|c|}
\hline xTerms_Online & handwriting \\
\hline
\end{tabular}

\section{INTRODUCTION}

Due to the tremendous progress in pattern recognition technology, handwriting-based human-computer-interaction (HCI) has become an indispensable component in our daily life. According to input signals, handwritten character recognition can be divided into online and offline recognition. Online recognition recognizes the stroke trajectories of handwritten characters, while offline recognition identifies the images of handwritten characters. Therefore, the input signals for online and offline recognition are the coordinate information of the pen tip as functions of time and the scanned image of the handwritten character, respectively. Since the online recognition can translate human's intentions to a computer more intuitively and effectively than the offline method, the input devices for the online handwriting recognition are widely developed in the last decade such as ultrasonic digital pens, infrared digital pens, and touch pads. However, the drawback of the abovementioned input devices is that they should be operated with ambit restrictions.

Recently, pen-based input devices embedded with inertial sensors have been proposed to sense or capture acceleration signals generated by writing trajectories. The inertial-sensor-based input devices can provide the coordinate information of the pen tip as functions of time for online handwriting recognition [1], [2]. A significant advantage of inertial-sensor-based input devices for handwriting recognition is that they can be operated without any external reference or ambit restrictions [1], [2], [3], [4]. Recently, many researchers have focused on the development of inertial-sensor-based input device. To name a few, Wang and Chuang [4] presented an accelerometerbased digital pen with a trajectory recognition algorithm for 2D handwritten digit recognition. The proposed algorithm extracted the time- and frequency-domain features from the accelerations, and then selected the most important features by the kernel-based class separability (KBCS) and linear discriminant analysis (LDA). Finally, a probabilistic neural network $(\mathrm{PNN})$ recognized the handwritten digits based on the reduced features and the recognition rate achieved at 98\%. Choi et al. [5] proposed a digital pen with a triaxial accelerometer to recognize $3 \mathrm{D}$ handwritten digits. They used the under-sampling and principle components analysis (PCA) methods to reduce the data size and the computation time. Subsequently, the hidden Markov model (HMM) was utilized to classify the movement signals. The userdependent and user-independent recognition rates using the HMM classifier were $100 \%$ and $68.6 \%$, respectively. Zhou et al. [6] developed a micro inertial measurement unit ( $\mu \mathrm{IMU})$ composed of an accelerometer and a gyroscope to recognize $2 \mathrm{D}$ handwritten digits and $2 \mathrm{D}$ handwritten English letters. The recognition rates of the handwritten digits and English letters were about $85 \%$ and $64 \%$ by using the self-organizing map (SOM) classifier. The abovementioned results show that HMMs and NNs are effective in dealing with handwriting recognition problems. However, HMMs and NNs both consume more computational time to obtain acceptable recognition rates in the training stage.

In this paper, we present an accelerometer-based pen device with an automatic recognition algorithm for $3 \mathrm{D}$ handwritten digit recognition. The pen device is composed of a triaxial accelerometer, a microcontroller, and an RF wireless transmission module. Users can utilize this accelerometer-based pen device to write digits in a 3-D space at normal speed. The automatic recognition algorithm is performed in a personal computer (PC) for recognizing the accelerations which are measured by the triaxial accelerometer and then transmitted from the pen device via the wireless module. The proposed automatic recognition procedure is composed of signal acquisition, signal 
preprocessing, and DTW recognizer. The advantages of the proposed pen device and its associated recognition strategy include the following: 1) with the accelerometer-based pen device, users can use the pen device to deliver their intentions by hand motions to remote control electronics devices anywhere without space limitations, and 2) the DTW recognizer requires less computational time than HMMs and NNs for handwriting recognition with high recognition accuracy.

The rest of this paper is organized as follows. In Section II, we introduce the hardware components of the accelerometer-based pen device in detail. The proposed automatic recognition algorithm consisting of signal acquisition, signal preprocessing, and DTW recognizer is presented in Section III. In Section IV, experimental results are presented to validate the proposed approach. Finally, conclusions are given in the last section.

\section{ACCelerometer-BAsed Pen DeVice}

The proposed accelerometer-based pen device is composed of a triaxial accelerometer (LSM303DLH, STMicroelectronics), a microcontroller (STM32F103T8, STMicroelectronics) and an RF wireless transceiver (nRF2401, Nordic). The accelerometer is used to detect accelerations of hand motions and its sensitivity was set from $-4 \mathrm{~g}$ to $+4 \mathrm{~g}$ in this paper. The microcontroller collects the digital signals generated from the accelerometer and wirelessly transmits the data to a $\mathrm{PC}$ main processor for further signal processing and recognition via the RF wireless transceiver. The sampling rate of the microcontroller is $100 \mathrm{~Hz}$. The pen-type input device is shown as Fig. 1 and its size is $130 \mathrm{~mm} \times 15 \mathrm{~mm} \times 8 \mathrm{~mm}$. The overall power consumption of the input device is 30 $\mathrm{mA}$ at $3.7 \mathrm{~V}$.

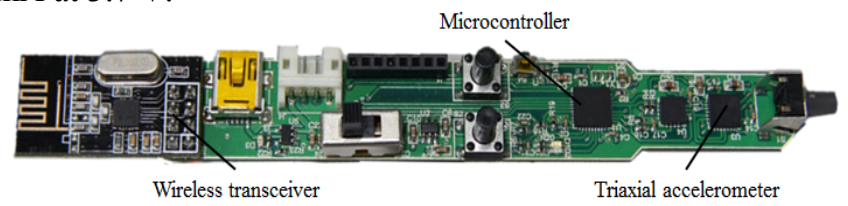

Fig. 1. Accelerometer-based input device.

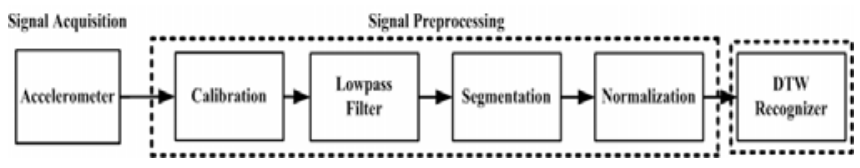

Fig. 2. Block diagram of the recognition algorithm.

\section{Automatic ReCOGNition Algorithm}

An automatic recognition algorithm has been developed to perform $3 \mathrm{D}$ handwritten digit recognition task using the acceleration signals measured by the triaxial acceleromater embeeded in the input device. The proposed automatic recognition algorithm shown in Fig. 2 consists of the following procedures: 1) signal acquisition, 2) signal preprocessing, and 3) DTW recognizer. We now introduce the detailed procedures of the proposed automatic recognition algorithm as follows.

\section{A. Signal Acquisition and Preprocessing}

In this paper, the acceleration signals of the hand motions are measured by a triaxial accelerometer. At the beginning of the procedure, a PC receives the accelerations via the RF transceiver. Subsequently, signal preprocessing is used to eliminate the intrinsic error sources of the accelerometer and users' unconscious trembles. The signal preprocessing procedure is composed of calibration, a lowpass filter, segmentation, and normalization. Firstly, we need to calibrate the accelerometer to reduce its errors of sensitivity and offset before using the accelerometer-based pen device. Secondly, we utilize a lowpass filter to remove high-frequency noise from the raw data. Then, an adaptive magnitude threshold is used to segment the acceleration patterns of hand movements when writing numerals in a 3-D space. Finally, the amplitude of each acceleration pattern has been normalized to the interval $[0,1]$ to avoid extreme amplitude scaling.

Calibration: To perform the calibration of the triaxial accelerometer, we place it on a leveled surface and then align each axis with the Earth's gravity as shown in Fig. 3. When the pen device is stationary, the triaxial accelerometer measures only the gravitational acceleration. Then, we can obtain the scale factor (SF) and bias (B) in each axis of the accelerometer from the measurements as follows.

$$
\begin{gathered}
S F=\frac{V(+g)-V(-g)}{2 g}, \\
B=\frac{V(+g)+V(-g)}{2},
\end{gathered}
$$

where $\mathrm{V}(+\mathrm{g})$ and $\mathrm{V}(-\mathrm{g})$ are the voltage outputs in each axis of the accelerometer when it is aligned with the direction of the Earth's gravity and with the opposite direction of Earth's gravity, respectively. Once the scale factor (SF) and bias (B) in each axis of the accelerometer can be found, we can use the following equation to calculate the calibrated accelerometer measurements.

$$
A_{c}=\frac{V-B}{S F},
$$

where $\mathrm{V}$ is the output voltage in each axis of the accelerometer. The more detailed information about the calibration can be found in [2].

Lowpass Filter: After we obtain the calibrated measurements (Ac) from the above calibration process, a moving average filter is used to reduce the high frequency noise of the calibrated accelerations, and the filter is expressed as

$$
A_{f}[n]=\frac{1}{N} \sum_{i=1}^{N} A_{c}[n-i],
$$

where $A c[n]$ is the calibrated accelerations, $\operatorname{Af}[n]$ is the filtered accelerations, and $\mathrm{N}$ is the number of points in the average filter ( $\mathrm{N}=7$ is used in this paper). 
Segmentation: We segment the motion intervals of all filtered acceleration patterns of hand movements to obtain accurate locations of the start and end points of each pattern based on an adaptive magnitude threshold. The adaptive magnitude threshold is defined as the mean of the filtered accelerations collected by keeping the accelerometer stationary in the beginning.

$$
T H=\operatorname{mean}_{k}\left(\left|\sqrt{A_{f x}^{2}(k)+A_{f y}^{2}(k)+A_{f z}^{2}(k)}-1\right|\right),
$$

where $\mathrm{k}$ denotes the time steps. The start and end points of the motion interval can be determined when the magnitudes are higher and lower than the threshold, respectively.

Normalization: We normalize each segmented acceleration pattern into the interval $[0,1]$ via the following equation:

$$
A_{\text {norm }}=\frac{A-\min (A)}{\max (A)-\min (A)},
$$

where $\max (\mathrm{A})$ and $\min (\mathrm{A})$ is the maximum and minimum amplitude among the segmented acceleration signals of the three axes in the motion interval, respectively. Anorm presents the normalized acceleration signals in the motion interval.
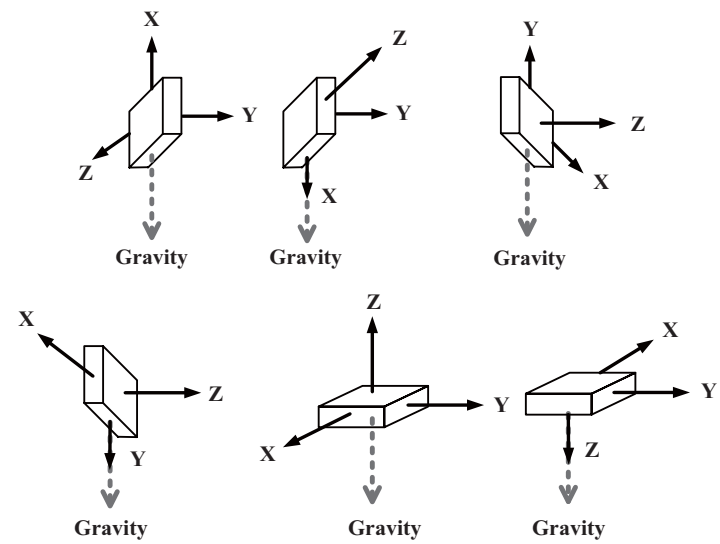

Fig. 3. Six cases of the triaxial accelerometer with one axis parallel to the Earth's gravity vector for accelerometer calibration.

\section{B. DTW Recognizer}

The Dynamic time warping (DTW) algorithm looks for an optimal alignment, which ensures a minimized cumulative distance measurement between the aligned sequences, to find the similarity between two time temporal sequences. Let $\mathrm{H}$ and $\mathrm{R}$ be two similar time sequences with the same sampling rate and different lengths, where $\mathrm{H}=[\mathrm{h} 1$, $h 2, \ldots, h m]$ and $R=[r 1, r 2, \ldots, r n]$. The optimal warping path can be found effectively based on dynamic programming by using the following formulation:

$$
D_{p, q}=d\left(h_{p}, r_{q}\right)+\min \left\{D_{p, q-1}, D_{p-1, q}, D_{p-1, q-1}\right\},
$$

where $d\left(h_{p}, r_{q}\right)=\sqrt{\left(h_{p}-r_{q}\right)^{2}}$ is the Euclidean distance, which is used to measure local distances between the two time sequences. The cumulative distance $\operatorname{DTW}(\mathrm{H}, \mathrm{R})$ between the two time sequences is then calculated by summing the local distances over the optimal warping path and can be defined as follows.

$$
\operatorname{DTW}(\mathbf{H}, \mathbf{R})=D_{m, n} .
$$

The more detailed information about the DTW algorithm can be found in [7].

The recognition performance greatly depends on the quality of the selected class templates of the DTW recognizer. Hence, to select reliable training class templates for each class from all templates within the same class to be recognized is the most important task in the training stage of the DTW recognizer. In this paper, the minimum selection method presented by [8] is used to perform the template selection task. At first, the sum of the DTW distance between the template and all other patterns within the same class is calculated. Then, the pattern with the minimum intra-class DTW distance is selected as the class template. In other words, there is only one class template for each digit by using the DTW recognizer. Finally, in the testing stage of the DTW recognizer, the recognition results are outputted through the DTW recognizer by measuring the similarity between the testing data and the selected class templates. Since the accelerations of each digit is composed of three acceleration waveforms (X-, Y-, and Z-axis), the similarity between the class template $\mathrm{C} \in \mathbb{R} \mathrm{m} \times 3$ and the testing acceleration pattern $T \in \mathbb{R} n \times 3$ can be computed as

$$
\operatorname{DTW}(\mathbf{C}, \mathbf{T})=\sqrt{D_{m, n}^{2}(x)+D_{m, n}^{2}(y)+D_{m, n}^{2}(z)},
$$

where $\operatorname{Dm}, \mathrm{n}(\mathrm{x}), \operatorname{Dm}, \mathrm{n}(\mathrm{y}), \operatorname{Dm}, \mathrm{n}(\mathrm{z})$ are the DTW cumulative distance computed between the traces in the X-, Y-, and Z-axis, respectively. Finally, the minimal DTW(C,T) representing that the testing sequence $\mathrm{Tn}$ and the class template $\mathrm{Cm}$ are within the same class.

\section{EXPERIMENTAL RESULTS}

In this section, the effectiveness of the proposed accelerometer-based pen device and its associated automatic recognition algorithm were validated by the $3 \mathrm{D}$ handwritten digit recognition experiment. The proposed automatic recognition algorithm consists of the following procedures: signal acquisition, signal preprocessing, and DTW recognizer. We collected the acceleration signals of this experiment from ten subjects ( 3 females, 7 males; age 23.5 \pm 2.01 years old) in a laboratory environment. The digital output signals of the accelerometer are all sampled at 100 Hz. Our experiment was performed on a PC running Microsoft Windows 7 operating system with an Intel ${ }^{\circledR}$ Core Processor i5-2400 and 8-GB RAM.

\begin{tabular}{|c|c|c|c|c|c|c|c|c|c|}
\hline 0 & 1 & 2 & 3 & 4 & 5 & 6 & 7 & 8 & 9 \\
\hline & $\ddots$ & $\ddots$ & $\ddots$ & $\square$ & $\ddots$ & $\ddots$ & 5 & $?$ \\
\hline
\end{tabular}

Fig. 4. Pictorial digit trajectories. 
TABLE I

USER-INDEPENDENT CONFUSION MATRIX FOR 3D HANDWRITTEN DIGIT RECOGNITION

\begin{tabular}{c|cccccccccc}
\multicolumn{10}{c}{ RECOGNITION } \\
\hline $\mathrm{W}$ & 0 & 1 & 2 & 3 & 4 & 5 & 6 & 7 & 8 & 9 \\
\hline 0 & 89 & 0 & 11 & 0 & 0 & 0 & 0 & 0 & 0 & 0 \\
1 & 0 & 100 & 0 & 0 & 0 & 0 & 0 & 0 & 0 & 0 \\
2 & 6 & 0 & 94 & 0 & 0 & 0 & 0 & 0 & 0 & 0 \\
3 & 2 & 0 & 1 & 88 & 0 & 0 & 0 & 0 & 9 & 0 \\
4 & 0 & 0 & 0 & 0 & 85 & 9 & 0 & 0 & 6 & 0 \\
5 & 0 & 10 & 0 & 0 & 0 & 90 & 0 & 0 & 0 & 0 \\
6 & 0 & 19 & 0 & 0 & 0 & 0 & 70 & 0 & 11 & 0 \\
7 & 0 & 6 & 0 & 0 & 0 & 0 & 0 & 94 & 0 & 0 \\
8 & 4 & 0 & 0 & 11 & 0 & 0 & 13 & 4 & 68 & 0 \\
9 & 0 & 30 & 0 & 0 & 0 & 0 & 0 & 0 & 0 & 70 \\
\hline \hline
\end{tabular}

R: Recognized, W: Written

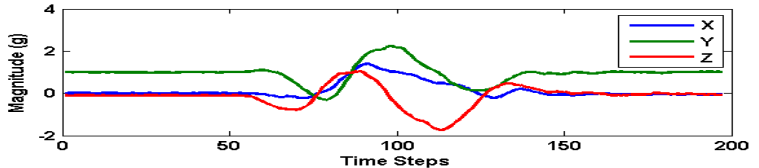

(a)

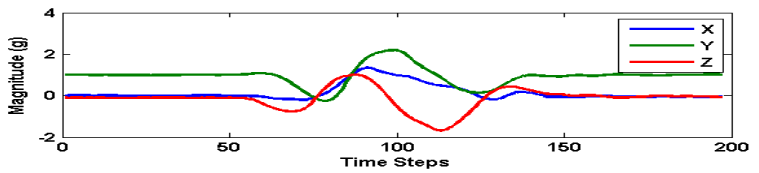

(b)

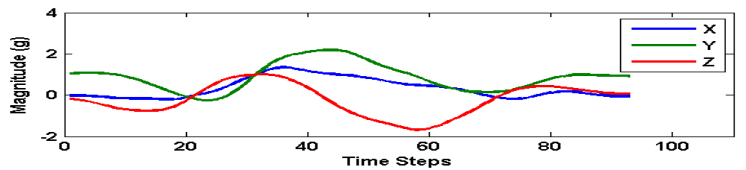

(c)

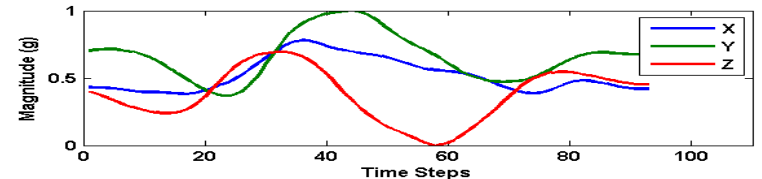

(d)

Fig. 5. Acceleration at each steps of the signal preprocessing for the digit 0 . (a) Calibrated accelerations. (b) Filtered accelerations. (c) Segmented accelerations. (d) Normalized accelerations.

In this experiment, the ten participants were asked to hold the pen device to write ten digits, which are shown in Fig. 4, without any ambit restriction in a 3-D space. Each participant was asked to repeat each digit for ten times. Therefore, a total of $1000(=10 \times 10 \times 10)$ data were generated. The acceleration signals in the experiment in the signal preprocessing procedure of the proposed automatic recognition algorithm for digit 0 are shown in Fig. 5. The calibrated and filtered accelerations via the calibration and filtering procedures are shown in Fig. 5(a) and Fig. 5(b), respectively. Subsequently, Fig. 5(c) shows the segmented accelerations in the motion interval. Finally, the normalized accelerations in the motion interval are shown in Fig. 5(d). Once all normalized acceleration signals in the motion intervals were acquired, the DTW recognizer chose the optimal templates for each digit class. The overall userdependent and user-independent recognition rates were $90.6 \%$ and $84.8 \%$, respectively. The user-independent confusion matrix for 3D handwritten digit recognition using the accelerometer-based pen device with the proposed automatic recognition algorithm is shown in Table I. The possible cause for misrecognition is that the temporal accelerations between the digits were pretty similar and different to recognize by using the DTW recognizer.

\section{CONCLUSION}

This paper has proposed an accelerometer-based pen device and its associated automatic recognition algorithm for $3 \mathrm{D}$ online handwritten digit recognition. The automatic recognition algorithm includes signal acquisition, signal preprocessing, and DTW recognizer. To improve the performance of the DTW recognizer, all acceleration patterns are normalized into the interval $[0,1]$ and the class template is selected via the minimum selection method. In the 3D handwritten digit recognition experiment, a total of 1000 digits with a set of 10 numerals from 10 users were used to validate the effectiveness of the proposed pen device and algorithm. The overall user-dependent and userindependent recognition rates were $90.6 \%$ and $84.8 \%$, respectively. Such results encourage us to further investigate the possibility of using our accelerometer-based pen device as an effective tool for HCI applications.

\section{REFERENCES}

[1] W. C. Bang, W. Chang, K. H. Kang, E. S. Choi, A. Potanin, and D. Y. Kim, "Self-contained spatial input device for wearable computers," in Proc. IEEE Int'l Conf. Wearable Computers, pp. 26-34, 2003.

[2] J. S. Wang, Y. L. Hsu, and J. N. Liu, "An inertialmeasurement-unit-based pen with a trajectory reconstruction algorithm and its applications," IEEE Trans. Industrial Electronics, vol. 57, no. 10, pp. 3508-3521, 2010.

[3] J. K. Oh, S. J. Cho, W. C. Bang, W. Chang, E. Chio, J. Yang, J. Cho, and D. Y. Kim, "Inertial sensor based recognition of 3-D character gestures with an ensemble of classifiers," in Proc. IEEE 9th Int. Workshop Frontiers Handwriting Recognit., 2004, pp. 112-117.

[4] J. S. Wang and F. C. Chuang, "An accelerometer-based digital pen with a trajectory recognition algorithm for handwritten digit and gesture recognition," IEEE Trans. Industrial Electronics, vol. 59, no. 7, pp. 2998-3007, 2011.

[5] S. D. Choi, A. S. Lee, and S. Y. Lee, "On-line handwritten character recognition with $3 \mathrm{D}$ accelerometer," in IEEE Int'l Conf. Information Acquisition, pp. 845-850, 2006.

[6] H. Zhou, T. Stone, H. Hu, and N. Harris, "Use of multiple wearable inertial sensors in upper limb motion tracking," Medical Engineering \& Physics, vol. 30, no.1, pp. 123-133, 2008.

[7] A. Akl, C. Feng, and S. Valaee, "A novel accelerometer-based gesture recognition system," IEEE Trans. Signal Processing, vol. 59, no. 12, pp. 6197-6205, 2011.

[8] M. H. Ko, G. West, S. Venkatesh, and M. Kumar, "Using dynamic time warping for online temporal fusion in multisensory systems," Information Fusion, vol. 9, no. 3, pp. 370-388, 2010. 